\title{
In-field experimental stress analysis in the elastic and plastic fields on motorbike handlebar clamped joints
}

\author{
G. Olmi ${ }^{1, a}$
}

${ }^{1}$ DIEM Department, University of Bologna, Viale del Risorgimento 2, 40136 Bologna, Italy

In a motorbike different types of shaft-hub couplings are used to join the handlebar to the suspension legs of the front wheel. Some papers were recently published with reference to the experimental determination of the starting friction coefficient of the fork-pin compression coupling [1], to the development of FEM assisted design strategies for the fork-pin [2], fork-leg [3] and leg-pin couplings [4]. On the other hand, another important joint, the clamping between the fork and the handlebar has been less investigated. The clamp (aluminum alloy, EN AW-6082, Figure 1A, B) consists of two parts, the lower one (connected to the fork by two screws), interfacing with the lower part of the handlebar, and the upper one, shaped to lodge the upper part of the handlebar. The assembly procedure consists in the insertion of the handlebar between the two clamp parts and in screw tightening, according to a particular procedure. Screws indicated as 1 and 2 are tightened first to create contact between mating surfaces of the two clamp parts, screws 3 and 4 are then applied to complete clamp closing.
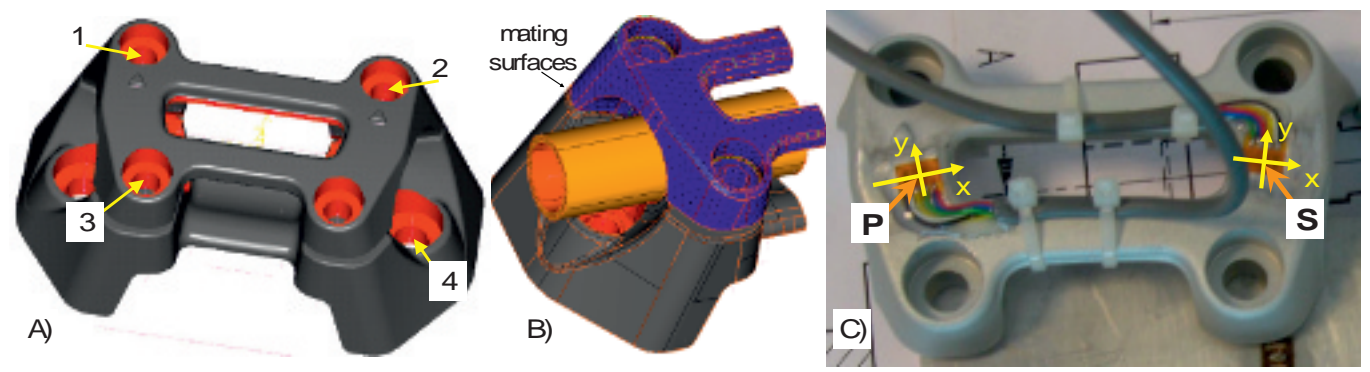

Fig. 1. Upper and lower part of the handlebar clamped joint (A, B), strain gage rosettes at locations $P$ and $S$ (C).

No information are available about stress trends during screw tightening, and about non symmetric effects in stress distribution, depending on the order of tightening of screws 3 and 4 . Moreover, no studies are available on the effects of yield strength overcome over a small region, and in case, on the safety of disassembling and reassembling the same clamp during maintenance procedures. Consequently, the main objects of this work are the followings:

- Experimental determination of stress trends in the elastic field: discussion on non symmetric effects depending on tightening sequence.

- Experimental stress analysis in the elasto-plastic field and discussion on the safety of the coupling, even in case of disassembling and reassembling of the same parts

The first step consisted in the execution of a FEM analysis of the coupling clamp-handlebar. For this purpose, a linear simulation was performed by I-DEAS $11 \mathrm{NX}$ with solid tetrahedral elements,

a e-mail : giorgio.olmi@unibo.it

This is an Open Access article distributed under the terms of the Creative Commons Attribution-Noncommercial License 3.0, which permits unrestricted use, distribution, and reproduction in any noncommercial medium, provided the original work is properly cited. 
and with the use of hitting and target contact regions for modelling the contact between the upper and the lower part of the clamp, and between these two parts and the handlebar. Two different couplings were modelled, with similar geometries for the clamps, but with different materials and characteristics for the handlebar. For type I the handlebar is in Aluminium alloy, while for type II it is in steel with knurled contact surfaces. The model led to the determination of the most stressed regions, referenced as zones $\mathrm{P}$ and $\mathrm{S}$ in Figure 1C. $0^{\circ} / 90^{\circ}$ Strain gauge rosettes (062UT, Vishay Intertechnology, Inc., Malvern, PA, USA) were applied along the principal directions and experimental tests were performed with simultaneous analog acquisition of grid readings and of the twisting moment applied by a torque wrench. A maximum torque corresponding to $40 \%$ of its nominal value (the value used according to the standard tightening procedure), the same used for FEM simulation, was initially considered for the first tests in order to maintain the stress state in the elastic field. Coupling types I and II were involved in the tests and two different tightening sequences (1-2-3-4 and 1-2-4-3) were considered. By comparing Von Mises stresses in regions $\mathrm{P}$ and $\mathrm{S}$, also by running ANOVA tests with six replications for each combination, it was showed that there are no significant differences in the induced stress distributions, so the two tightening procedures are interchangeable. The FEM model was calibrated and validated, by comparing experimental and numerical results with acceptable differences (5-10\% for type I and 10-20\% for type II).

Tests were then performed with the standard nominal tightening torque. In this case a plasticization was observed with residual strains after load removal: strains measured by strain gauges were processed according to a non linear model (Figure 2A, B). It is interesting to notice that for type II the maximum stress along y has a particular trend: it is initially negative, with a sudden sign change as the load is increased. It seems to be related to a stick-slip phenomenon, due to the knurled surface of the handlebar and to the sudden overcome of its static friction coefficient. Finally, the safety of coupling assembling and disassembling was studied, by implementing a numerical simulation of the stress-strain cycle in such procedures. Results showed that the cycle remains totally in the elastic field, i.e. there is no hysteresis, when the clamp is opened and closed again.

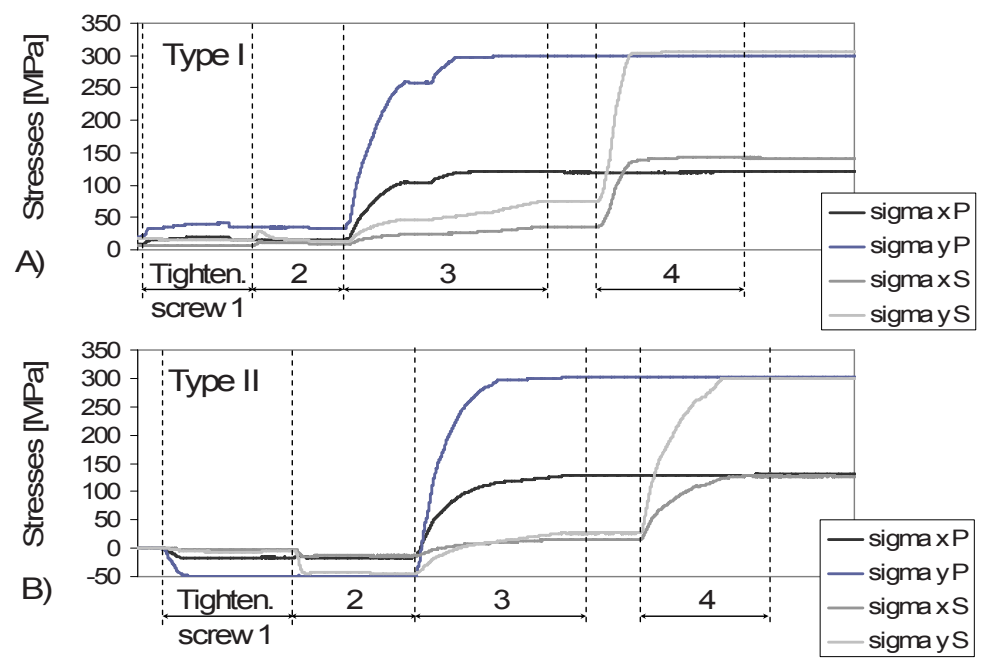

Fig. 2. Stress trends during tightening for types I (A) and II (B).

\section{References}

1. D. Croccolo, R. Cuppini, N. Vincenzi, Strain, 44, 170-179 (2008)

2. D. Croccolo, R. Cuppini, N. Vincenzi, Fin. El. in Analysis and Design, 43, 977-988 (2007)

3. D. Croccolo, R. Cuppini, N. Vincenzi, Proc. of IMECE 2008, 12, 169-178 (2009)

4. D. Croccolo, R. Cuppini, N. Vincenzi, Fin. El. in Analysis and Design, 45, 406-414 (2009) 